\title{
LOCALIZATION PERFORMANCE OF 1-BIT PASSIVE RADARS IN NB-IOT APPLICATIONS
}

\author{
Saeid Sedighi, Kumar Vijay Mishra, Bhavani Shankar M. R. and Björn Ottersten \\ Interdisciplinary Centre for Security, Reliability and Trust (SnT), University of Luxembourg \\ E-mail: \{saeid.sedighi@, kumar.mishra@ext., bhavani.shankar@, bjorn.ottersten@ \}uni.lu
}

\begin{abstract}
Location-based services form an important use-case in emerging narrowband Internet-of-Things (NB-IoT) networks. Critical to this offering is an accurate estimation of the location without overlaying the network with additional active sensors. The massive number of devices, low power requirement, and low bandwidths restrict the sampling rates of NB-IoT receivers. In this paper, we propose a novel low-complexity approach for NB-IoT target delay estimation in cases where one-bit analog-to-digital-converters (ADCs) are employed to sample the received radar signal instead of high-resolution ADCs. This problem has potential applications in the design of inexpensive NB-IoT radar and sensing devices. We formulate the target estimation as a multivariate fractional optimization problem and solve it via Lasserre's semi-definite program relaxation. Numerical experiments suggest feasibility of the proposed approach yielding high localization accuracy with a very low number of 1-bit samples.
\end{abstract}

Index Terms - Fractional optimization, localization, narrowband internet-of-things, one-bit quantization, passive radar.

\section{INTRODUCTION}

With the rapid proliferation in wireless and web-based services, the Internet of Things (IoT) is envisioned to connect the physical and digital world through extensive instrumentation of the former with sensing, wearable, and intelligent devices [1]. Since IoT framework is defined by a massive number of largely battery-powered devices, that rarely transmit or receive data, the underlying challenges for any communications link here are low power, low data rate, wide coverage and scalability [2]. In this context, the 3rd generation partnership project (3GPP) recently introduced narrowband (NB) IoT system specifications to support wide coverage area, long user lifetime, and low power/cost devices over a narrow bandwidth of $180 \mathrm{kHz}$ [3].

One of the most attractive IoT applications is localization-based service [4], wherein a large network of devices collects and transmits data in order to determine the position of targets-of-interest. A common localization technique is deployment of passive sensor tags that augment existing IoT deployments through backscatter communications [5]. This is a feasible approach given that it is difficult to repurpose the IoT network sensing modalities that are usually fixed before the deployment and comprise millions of devices [6]. Addition of passive sensors does not require changing the deployed IoT hardware or addition of new communications and power sources [7]. In this paper, we focus on the passive localization for NB-IoT.

While not fully backward compatible with existing 3GPP devices, the NB-IoT harmoniously coexists with legacy networks by reusing the functionalities of the latter's design. Since the NB-IoT

This work is supported by the National Research Fund, Luxembourg under AFR grant for Ph.D. project (Project Reference 11228830) on Compressive Sensing for Ranging and Detection in Automotive Applications. bandwidth is limited, its transmit power spectral density is enhanced within the existing 3GPP specifications. This, combined with a soft re-transmission strategy, enhances the coverage of NB-IoT over conventional IoT solutions [8]. The ultra-low complexity and low power consumption features of NB-IoT are advantageous for location-based services such as smart parking, smart tracking, and smart home [9]. At the same time, low NB-IoT bandwidth severely limits the data rate. The massive number of devices also render deployment of the Global Positioning System (GPS) device at every node very expensive. Finally, low battery-power of NB-IoT devices is insufficient to handle high sampling rates required to attain necessary localization accuracy [10-13]. These drawbacks are often compensated by employing advanced algorithms for NB-IoT localization [4].

A popular NB-IoT localization technique is to employ fingerprinting, wherein the received signal strength indicator (RSSI) measurements are collected at specified locations during the training phase and then compared with online measurements to determine the location of the target $[10,14]$. This approach requires prior knowledge of RSSI database. Some recent NB-IoT works also explore RSSI-independent signal processing methods such as successive interference cancellation [11], maximum likelihood estimation [12], and frequency hopping [13]. In this paper, we adopt a technique inspired by localization in passive radar arrays [15] that does not require prior RSSI measurements. Further, in order to combat the problem of achieving high accuracy under low bandwidths, we leverage recent advances in 1-bit signal processing $[16,17]$ in NB-IoT localization.

Ideally, the analog-to-digital conversion requires an infinite number of bits to accurately represent the continuous-time signal in the digital domain [18]. In practice, the signal is quantized to a finite number of bits leading to errors in digital approximation of the original analog signal. If the sampling resolution is large enough, this error has negligible effect on digital signal processing. However, in NB-IoT, the measurements are quantized to very low bit-rates and the high-precision data is rarely available. Further, the cost and power consumption of ADCs increases linearly with the number of quantization bits and sampling frequency [19]. Therefore, to support the low cost feature of NB-IoT, the number of quantization bits should be reduced. This necessitates development of algorithms for low-bit scenario which, in the extreme case, is just a single bit per sample.

Originally proposed $[16,20,21]$ in the context of compressive sensing [22], the 1-bit sampling has a rich heritage of research in statistical signal processing [23-26] and signal reconstruction [27, 28 ]. In the past few years, the 1-bit processing has received significant attention in numerous modern applications such as classical communications [29-31], massive multiple-input multiple-output (MIMO) [32, 33], deep learning [34], dictionary learning [35, 36], and radar [37-39]. In nearly most of these works, the approach is to repeatedly compare the signal of interest with a time-varying threshold reference [40]. For example, the single-bit sampling scheme in [41] records the positions of zero-crossings of the input signal 
added to a deterministic dither function. It was shown here that, for band-limited bounded-amplitude square-integrable input signal, sufficient number of one-bit samples lead to recovery of full-precision data with locally bounded point-wise error, resulting in an exponentially decaying distortion-rate characteristic.

In this paper, we model target localization using one-bit measurements obtained from passive sensors in NB-IoT systems. We cast the localization problem using bistatic range-difference model which results in a system of several equations solved conventionally by least squares (LS) method [15]. However, unlike the conventional case where infinite precision range measurements are available, we assume only 1-bit samples. We then provide a novel formulation of an optimization problem to jointly estimate the infinite precision data as well as the target location. Contrary to previous works on 1-bit radar [40], our proposed method solves the resulting optimization problem efficiently using the Lasserre's semi-definite program (SDP) relaxation [42] that benefits from a small feasibility set. To the best of our knowledge, ours is the first work that investigates 1-bit sampling in a passive radar array setting.

Throughout this paper, we refer the vectors and matrices by lowerand upper-case bold-face letters, respectively. The superscript $(\cdot)^{T}$ indicates the transpose operation. The notations $\|\mathbf{a}\|_{2}$ and $\hat{\mathbf{a}}$ denote $\ell_{2}$-norm and the estimate of $\mathbf{a}$, respectively. A diagonal matrix with the diagonal vector $\mathbf{a}$ is represented by $\operatorname{diag}(\mathbf{a})$. Further, $\mathbf{A}^{\dagger}$ and $\Pi_{\mathbf{A}}^{\perp}=\mathbf{A A}^{\dagger}$ indicate the pseudo-inverse and the projection matrix onto the null space of the full column rank matrix $\mathbf{A}$, respectively; whereas the null space of $\mathbf{A}$ is $\mathcal{N}(\mathbf{A})$. When all elements of a vector $\mathbf{a}$ are greater than or equal to zero, we indicate this as $\mathbf{a} \succeq \mathbf{0}$. The symbol $\odot$ represents the Hadamard (element-wise) product and $\operatorname{sng}(\cdot)$ stands for the sign function.

\section{SYSTEM MODEL}

Consider a source, say a communications base station, transmits a known signal that is bounced off a target and the backscattered signal is received by $M$ different NB-IoT nodes, which are synchronized with the base station but are not synchronized with each other (Fig. 1). The $i^{\text {th }}$ sensor is located at the Cartesian coordinates $\left[x_{i}, y_{i}, z_{i}\right]^{T}, i \in\{1,2, \cdots, M\}$. The target is characterized by its location $[x, y, z]^{T}$. In a typical NB-IoT setting, a target could be a subject carrying a mobile phone, an intelligent vehicle or a robot.

We focus on the time-of-arrival (ToA) measurements to achieve the 3-dimensional localization using the measurements from these $M$ passive sensors. The ToA is defined as the one-way signal propagation time from the source to a sensor. Assuming the propagation is nondispersive, the ToA measured at $i^{\text {th }}$ sensor is given by

$$
\begin{gathered}
y_{i}(t)=s\left(t-\tau_{i}\right)+n_{i}(t), \\
t_{i}=\frac{d_{i}+\alpha}{c}=\underset{\tau}{\operatorname{argmin}} \int_{-\infty}^{\infty} y_{i}(t) s^{*}(t-\tau),
\end{gathered}
$$

where $c=3 \times 10^{8} \mathrm{~m} / \mathrm{s}$ is the speed of light in vacuum, $\alpha$ is the distance between the source and the target, $d_{i}$ is the distance between the target and the $i^{\text {th }}$ sensor described as

$$
d_{i}=\sqrt{\left(x_{i}-x\right)^{2}+\left(y_{i}-y\right)^{2}+\left(z_{i}-z\right)^{2}},
$$

where we assume ToA estimates are available accurately, i.e., in the absence of noise. Accordingly, the corresponding range is

$$
r_{i}=c t_{i}=d_{i}+c \alpha .
$$

The $i^{\text {th }}$ sensor converts its own range measurements into 1-bit data $\gamma_{i}$ after comparing it to an appropriate positive threshold $\lambda_{i}>0$ :

$$
\gamma_{i}=\operatorname{sng}\left(r_{i}-\lambda_{i}\right) .
$$

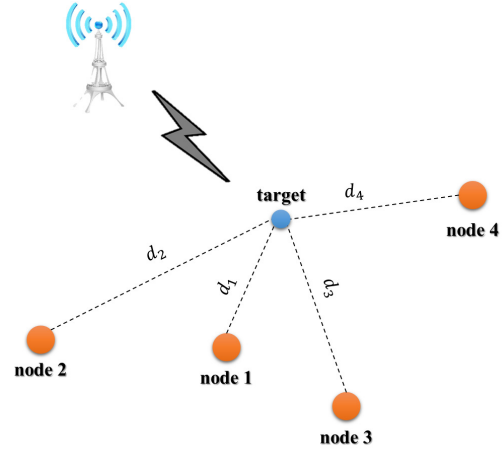

Fig. 1. Illustration of the localization scenario. The nodes represent passive sensors that receive the signal from the source bounced off from a target-of-interest.

These measurements are then forwarded to a fusion center where target localization takes place.

\subsection{Localization with infinite precision sampling}

Without loss of generality, consider the first sensor as the reference sensor. Computing the difference between the range measurements of $i^{\text {th }}$ and reference sensors yields

$$
\tilde{r}_{i}=r_{i}-r_{1}=d_{i}-d_{1},
$$

For the case of ideal (noiseless) ToA estimation,

$$
\tilde{r}_{i}+d_{1}=d_{i} \text {. }
$$

Squaring both sides of (7) and substituting $d_{i}$ from (3) produces the following system of equations, which are linear in $[x, y, z]^{T}$, $\left(x-x_{1}\right)\left(x_{i}-x_{1}\right)+\left(y-y_{1}\right)\left(y_{i}-y_{1}\right)+\left(z-z_{1}\right)\left(z_{i}-z_{1}\right)+\tilde{r}_{i} d_{1}$ $=\frac{1}{2}\left[\left(x_{i}-x_{1}\right)^{2}+\left(y_{i}-y_{1}\right)^{2}+\left(z_{i}-z_{1}\right)^{2}-\tilde{r}_{i}^{2}\right]$.

Collecting all linear equations specified by (8) for $i \in 1,2, \cdots, M$, we obtain the following compact matrix form

$$
\mathbf{G} \boldsymbol{\theta}=\mathbf{h},
$$

where

$\mathbf{G}=\left[\begin{array}{cccc}\left(x_{2}-x_{1}\right) & \left(y_{2}-y_{1}\right) & \left(z_{2}-z_{1}\right) & \tilde{r}_{2} \\ \vdots & \vdots & \vdots & \vdots \\ \left(x_{M}-x_{1}\right) & \left(y_{M}-y_{1}\right) & \left(z_{M}-z_{1}\right) & \tilde{r}_{M}\end{array}\right] \in \mathbb{R}^{(M-1) \times 4}$,

$$
\mathbf{h}=\frac{1}{2}\left[\begin{array}{c}
\left(x_{2}-x_{1}\right)^{2}+\left(y_{2}-y_{1}\right)^{2}+\left(z_{2}-z_{1}\right)^{2}-\tilde{r}_{2}^{2} \\
\vdots \\
\left(x_{M}-x_{1}\right)^{2}+\left(y_{M}-y_{1}\right)^{2}+\left(z_{M}-z_{1}\right)^{2}-\tilde{r}_{M}^{2}
\end{array}\right],
$$

and $\boldsymbol{\theta}=\left[x-x_{1}, y-y_{1}, z-z_{1}, d_{1}\right]^{T}$.

Even in case of infinite precision quantization, various non-ideal conditions such as receiver noise leads to perturbations in ToA estimate. We consider a simplified model, where these perturbations denoted by $\epsilon$, are related to ToA measurements as

$$
\epsilon=\mathbf{G} \boldsymbol{\theta}-\mathbf{h} \text {. }
$$

Solving (12) provides meaningful results when the range measurements $\tilde{r}_{i}$, for $i \in\{1,2, \cdots, M\}$, at the fusion center faithfully 
represent those at the sensors. Then, the LS solution provides an estimation of the target location as

$$
\hat{\boldsymbol{\theta}}=\mathbf{G}^{\dagger} \mathbf{h} \text {. }
$$

In a 1-bit sampling scenario, the actual (full precision) values of range measurements are not available. Instead, the fusion centre receives only one-bit samples, i.e., $\gamma_{1}, \cdots, \gamma_{M-1}$ wherein LS method is inapplicable. Our goal is to recover both range measurements and the target location with one-bit samples.

\section{ONE-BIT TOA FORMULATION}

Define $\tilde{\mathbf{r}}=\left[\begin{array}{llll}\tilde{r}_{2} & \tilde{r}_{2} & \cdots & \tilde{r}_{M}\end{array}\right]^{T} \in \mathbb{R}^{(M-1) \times 1}$. We rewrite $\mathbf{G}$ and $\mathbf{h}$ as

$$
\begin{aligned}
\mathbf{G} & =\left[\begin{array}{ll}
\mathbf{A} & \tilde{\mathbf{r}}
\end{array}\right], \\
\mathbf{h} & =\tilde{\mathbf{h}}-\frac{1}{2} \tilde{\mathbf{r}} \odot \tilde{\mathbf{r}},
\end{aligned}
$$

where

$$
\mathbf{A}=\left[\begin{array}{cccc}
\left(x_{2}-x_{1}\right) & \left(y_{2}-y_{1}\right) & \left(z_{2}-z_{1}\right) & \\
\vdots & \vdots & \vdots & \vdots \\
\left(x_{M}-x_{1}\right) & \left(y_{M}-y_{1}\right) & \left(z_{M}-z_{1}\right)
\end{array}\right] \in \mathbb{R}^{(M-1) \times 3}
$$

and

$$
\tilde{\mathbf{h}}=\frac{1}{2}\left[\begin{array}{c}
\left(x_{2}-x_{1}\right)^{2}+\left(y_{2}-y_{1}\right)^{2}+\left(z_{2}-z_{1}\right)^{2} \\
\vdots \\
\left(x_{M}-x_{1}\right)^{2}+\left(y_{M}-y_{1}\right)^{2}+\left(z_{M}-z_{1}\right)^{2}
\end{array}\right] \in \mathbb{R}^{(M-1) \times 1} .
$$

From the above, the LS objective is a function of both the desired target location and the full precision range measurements. We reformulate the LS cost function as

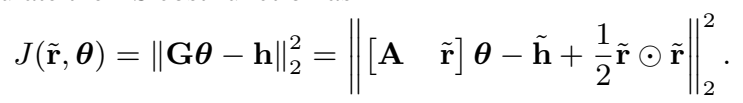

The solution of LS objective with respect $\boldsymbol{\theta}$ for a given $\tilde{\mathbf{r}}$ is given in (13). Substituting (13) into (17) yields

$$
\begin{aligned}
J(\tilde{\mathbf{r}}) & =J(\tilde{\mathbf{r}}, \hat{\boldsymbol{\theta}})=\left\|\Pi_{\mathbf{G}}^{\perp} \mathbf{h}\right\|_{2}^{2} \\
& =\left\|\left(\Pi_{\mathbf{A}}^{\perp}-\Pi_{\Pi_{\mathbf{A}}^{\perp} \tilde{\mathbf{r}}}\right)\left(\tilde{\mathbf{h}}-\frac{1}{2} \tilde{\mathbf{r}} \odot \tilde{\mathbf{r}}\right)\right\|_{2}^{2},
\end{aligned}
$$

where the last equality in (19) is obtained by making use of the projection decomposition theorem [43]. Since $\Pi_{\mathbf{A}} \frac{1}{\mathbf{r}} \in \mathcal{N}\left(\mathbf{A}^{H}\right)$, it is easily confirmed that $\Pi_{\mathbf{A}}^{\perp} \Pi_{\Pi_{\mathrm{A}}} \mathbf{\mathbf { r }}=\Pi_{\Pi_{\mathbf{A}}} \frac{1}{\mathbf{r}}$. Hence, (19) is simplified as,

$$
J(\tilde{\mathbf{r}})=\left(\tilde{\mathbf{h}}-\frac{1}{2} \tilde{\mathbf{r}} \odot \tilde{\mathbf{r}}\right)^{T}\left(\Pi_{\mathbf{A}}^{\perp}-\Pi_{\Pi_{\mathrm{A}}^{\perp} \tilde{\mathbf{r}}}\right)\left(\tilde{\mathbf{h}}-\frac{1}{2} \tilde{\mathbf{r}} \odot \tilde{\mathbf{r}}\right) .
$$

Considering the fact that

$$
\Pi_{\Pi_{\mathbf{A}}^{\perp} \tilde{\mathbf{r}}}=\frac{\Pi_{\mathbf{A}}^{\perp} \tilde{\mathbf{r}} \tilde{\mathbf{r}}^{T} \Pi_{\mathbf{A}}^{\perp}}{\tilde{\mathbf{r}}^{T} \Pi_{\mathbf{A}}^{\perp}, \tilde{\mathbf{r}}},
$$

we have $J(\tilde{\mathbf{r}})=\frac{f(\tilde{\mathbf{r}})}{g(\tilde{\mathbf{r}})}$ where

$$
\begin{aligned}
f(\tilde{\mathbf{r}})= & \left\|\Pi_{\mathbf{A}}^{\perp} \tilde{\mathbf{r}}\right\|^{2}\left[\left\|\Pi_{\mathbf{A}}^{\perp} \tilde{\mathbf{h}}\right\|^{2}+\frac{1}{4}\left\|\Pi_{\mathbf{A}}^{\perp}(\tilde{\mathbf{r}} \odot \tilde{\mathbf{r}})\right\|^{2}-\tilde{\mathbf{h}}^{T} \Pi_{\mathbf{A}}^{\perp}(\tilde{\mathbf{r}} \odot \tilde{\mathbf{r}})\right] \\
& -\left(\tilde{\mathbf{h}}^{T} \Pi_{\mathbf{A}}^{\perp} \tilde{\mathbf{r}}\right)^{2}-\frac{1}{4}\left((\tilde{\mathbf{r}} \odot \tilde{\mathbf{r}})^{T} \Pi_{\mathbf{A}}^{\perp} \tilde{\mathbf{r}}\right)^{2} \\
& +\tilde{\mathbf{h}}^{T} \Pi_{\mathbf{A}}^{\perp} \tilde{\mathbf{r}} \tilde{\mathbf{r}}^{T} \Pi_{\mathbf{A}}^{\perp}(\tilde{\mathbf{r}} \odot \tilde{\mathbf{r}}), \\
g(\tilde{\mathbf{r}})= & \left\|\Pi_{\mathbf{A}}^{\perp} \tilde{\mathbf{r}}\right\|^{2} .
\end{aligned}
$$

Note that $f(\cdot), g(\cdot)$ are multivariate polynomial functions of $\tilde{\mathbf{r}}$. It follows from $\tilde{r}_{i}=r_{i}-r_{1} \forall i$ that $f(\cdot), g(\cdot)$ are multivariate polynomial functions of $\mathbf{r}=\left[\begin{array}{llll}r_{1} & r_{2} & \cdots & r_{M}\end{array}\right]^{T} \in \mathbb{R}^{M \times 1}$ as well. In consequence, the joint estimation problem of finding $\boldsymbol{\theta}$ and $\tilde{\mathbf{r}}$ reduces to

$$
\begin{array}{ll}
\underset{\mathbf{r}}{\operatorname{minimize}} & \frac{f(\mathbf{r})}{g(\tilde{\mathbf{r}})} \\
\text { subject to } & \boldsymbol{\Gamma}(\mathbf{r}-\boldsymbol{\lambda}) \succeq \mathbf{0}, \\
& \mathbf{r} \succeq \mathbf{0} .
\end{array}
$$

where $\boldsymbol{\Gamma}=\operatorname{diag}(\boldsymbol{\gamma})$ with $\boldsymbol{\gamma}=\left[\begin{array}{lll}\gamma_{1}, \gamma_{2} & \cdots & \gamma_{M}\end{array}\right]^{T} \in\{0,1\}^{M \times 1}$.

The above formulation is a fractional optimization problem solving which yields $\mathbf{r}$. and, subsequently, the target location estimate via (13).

\section{ESTIMATION VIA LASSERRE'S SDP RELAXATION}

In order to solve the fractional optimization in (24), we reformulate it as an equivalent polynomial optimization problem. Then, we derive a smaller feasible set for this equivalent problem so that it is solved efficiently by exploiting the Lasserre's SDP relaxation method [42].

Theorem 1. The optimization problem in (24) is equivalent to the following problem

$$
\begin{aligned}
& \underset{\mathbf{r}, u}{\operatorname{minimize}} u^{6} f(\mathbf{r} / u) \\
& \text { subject to } u^{4} g(\mathbf{r})=1, \\
& \\
& \boldsymbol{\Gamma}(\mathbf{r}-u \boldsymbol{\lambda}) \succeq \mathbf{0}, \\
& \mathbf{r} \succeq \mathbf{0}, \\
& u>0 .
\end{aligned}
$$

Proof. Let define the sets $\mathcal{K}$ and $\tilde{\mathcal{K}}$ as follows

$$
\begin{aligned}
& \mathcal{K}=\left\{\mathbf{r} \in \mathbb{R}_{\geq 0}^{M} \mid \boldsymbol{\Gamma}(\mathbf{r}-\boldsymbol{\lambda}) \succeq \mathbf{0}\right\}, \\
& \tilde{\mathcal{K}}=\left\{\mathbf{r} \in \mathbb{R}_{\geq 0}^{M}, u>0 \mid \boldsymbol{\Gamma}(\mathbf{r}-u \boldsymbol{\lambda}) \succeq \mathbf{0}\right\} .
\end{aligned}
$$

It is then possible to rewrite optimization problems (24) and (25) as

$$
\begin{aligned}
& p^{*}=\underset{\mathbf{r}}{\operatorname{minimize}} \frac{f(\mathbf{r})}{g(\mathbf{r})}, \\
& \text { subject to } \quad \mathbf{r} \in \mathcal{K} \text {, } \\
& \tilde{p}^{*}=\underset{\mathbf{r}, u}{\operatorname{minimize}} u^{6} f(\mathbf{r} / u) \text {, } \\
& \text { subject to } u^{4} g(\mathbf{r})=1 \\
& {\left[\begin{array}{ll}
\mathbf{r}^{T} & u
\end{array}\right]^{T} \in \tilde{\mathcal{K}},}
\end{aligned}
$$

where $p^{*}$ and $\tilde{p}^{*}$ denote the minimums of optimization problems (24) and (25), respectively. Further, let $\mathbf{r}^{*} \in \mathcal{K}$ and $\left[\begin{array}{cc}\mathbf{r}^{* T} & u^{*}\end{array}\right]^{T} \in \tilde{\mathcal{K}}$ stand for the minimizers of optimization problems (24) and (25), respectively.

We first show that $p^{*} \leq \tilde{p}^{*}$. It readily follows from $u^{*}>0$ that $\overline{\mathbf{r}}^{*} / u^{*} \in \mathcal{K}$. Hence, exploiting that fact that $u^{* 4} g\left(\overline{\mathbf{r}}^{*}\right)=1$, we have

$$
p^{*} \leq \frac{f\left(\overline{\mathbf{r}}^{*} / u^{*}\right)}{g\left(\overline{\mathbf{r}}^{*} / u^{*}\right)}=u^{* 6} f\left(\overline{\mathbf{r}}^{*} / u^{*}\right)=\tilde{p}^{*} .
$$

Therefore, to show $p^{*}=\tilde{p}^{*}$, we simply need to prove that $p^{*} \geq \tilde{p}^{*}$ as well. It is easily confirmed that $\left[\begin{array}{ll}\mathbf{r}^{* T} & 1\end{array}\right]^{T} \in \tilde{\mathcal{K}}$. Thus, resorting to $g\left(\mathbf{r}^{*}\right)=1$ yields

$$
\tilde{p}^{*} \leq f\left(\mathbf{r}^{*}\right)=\frac{f\left(\mathbf{r}^{*}\right)}{g\left(\mathbf{r}^{*}\right)}=p^{*}
$$

This concludes the proof. 
The problem (25) is a polynomial optimization problem which can be solved by applying the Lasserre's SDP relaxation [42]. However, applying the Lasserre's SDP relaxation directly to the optimization problem (25) may need the high order Lasserre's SDP relaxation which could render solving the optimization problem computationally inefficient. In this regard, to solve the problem (25) in a more efficient manner, we first obtain a smaller feasible set for the optimization problem (25) exploiting the KKT conditions. Then, we apply the Lasserre's SDP relaxation to the new problem.

Let $\left[\begin{array}{ll}\overline{\mathbf{r}}^{*} & u^{*}\end{array}\right]^{T}$ be a mininmizer of (25). Since the objective and constraint functions are differentiable, the KKT conditions hold at $\left[\begin{array}{ll}\mathbf{r}^{*} & u^{*}\end{array}\right]^{T}$. This implies that the Jacobbian matrix of the objective and constraint functions, denoted by $F(\overline{\mathbf{r}}, u) \in \mathbb{R}^{M \times 4}$, should be rank deficient at $\left[\begin{array}{ll}\overline{\mathbf{r}}^{*} & u^{*}\end{array}\right]^{T}$. Let denote the determinant variety of $F(\mathbf{r}, u)$ being rank deficient by

$$
\mathcal{G}=\left\{\left[\begin{array}{ll}
\mathbf{r}^{T} & u
\end{array}\right]^{T} \in \mathbb{R}_{\geq 0}^{M} \mid \operatorname{rank}(F(\mathbf{r}, u)) \leq 4\right\} .
$$

Then, it follows that $\left[\begin{array}{ll}\overline{\mathbf{r}}^{*} & u^{*}\end{array}\right]^{T} \in \mathcal{G} \cap \mathcal{M}$ with $\mathcal{M}$ being the feasible set obtained from the constraints set of the optimization problem (25). Hence, it is possible to minimize the objective function in (25) over the new smaller feasible set $\mathcal{G} \cap \mathcal{M}$ instead of $\mathcal{M}$. Before proceeding further, we first need to find a more tractable description for $\mathcal{G}$. It is well-known that if a matrix is singular, all its maximal minors should vanish [44]. Let $H_{I}$ be the maximal minor of $F(\mathbf{r}, u)$ corresponding to the rows whose indices are determined by the set $I \subset S \doteq\{1,2, \cdots, M\}$ where $|I|=4$. Then,

$$
\mathcal{G}=\left\{\left[\begin{array}{ll}
\mathbf{r}^{T} & u
\end{array}\right]^{T} \in \mathbb{R}_{\geq 0}^{M}\left|H_{I}=0, \forall I \subset S,\right| I \mid=4\right\} .
$$

It was shown in $[45,46]$ that (33) has only $4 M-15$ generating equations each of which is given by

$$
h_{p}(\mathbf{r}, u)=\sum_{i_{i}+i_{2}+i_{3}+i_{4}=p+9} H_{I},
$$

where $i_{i}, i_{2}, i_{3}, i_{4} \in S$. Hence,

$\mathcal{G}=$

$\left\{\left[\begin{array}{ll}\mathbf{r}^{T} & u\end{array}\right]^{T} \in \mathbb{R}_{\geq 0}^{M} \mid h_{p}(\mathbf{r}, u)=0, \forall p \in\{1,2, \cdots, 4 M-15\}\right\}$.

Accordingly, the optimization problem (25) is equivalent to

$$
\begin{aligned}
\underset{\mathbf{r}, u}{\operatorname{minimize}} & u^{6} f(\mathbf{r} / u) \\
\text { subject to } & u^{4} g(\mathbf{r})=1, \\
& h_{p}(\mathbf{r}, u)=0, \quad \forall p \in\{1,2, \cdots, 2 M-15\}, \\
& \boldsymbol{\Gamma}(\mathbf{r}-u \boldsymbol{\lambda}) \succeq \mathbf{0}, \\
& \mathbf{r} \succeq \mathbf{0}, \\
& u>0 .
\end{aligned}
$$

Now the above optimization can be solved by using the Lasserre's SDP relaxation. Since the optimization problem (36) has a smaller feasible set compared to (25), it will need a lower-order Lasserre's SDP relaxation compared to (25). Hence, efficiency significantly increases by making use of (36).

\section{EXPERIMENTS AND DISCUSSION}

We investigated the performance of our proposed method through numerical simulations. We compared the 1-bit performance with the infinite precision range measurements (denoted by $\infty$-precision). For all experiments, 10000 Monte Carlo trials were conducted for any given number of 1-bit samples. We used $4^{\text {th }}$-order Lasserres SDP

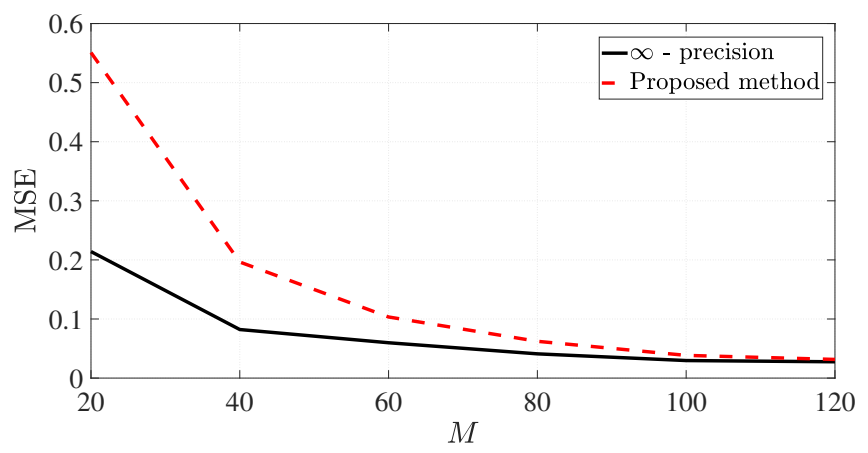

Fig. 2. The MSE in the estimate of target location with respect to the number of IoT devices, i.e., $M$.

relaxation to solve the optimization problem (25).

Figure 2 shows the mean-squared error (MSE) of the target location estimate versus the number of one-bit samples (the number of radar nodes), i.e., $M$, for both 1-bit and full-precision measurements. The MSE is defined as

$$
\left(\sum_{j=1}^{J}\left(x-\hat{x}_{j}\right)^{2}+\left(y-\hat{y}_{j}\right)^{2}+\left(z-\hat{z}_{j}\right)^{2}\right) / J,
$$

where $\left[\begin{array}{lll}\hat{x}_{j} & \hat{y}_{j} & \hat{z}_{j}\end{array}\right]^{T}$ denotes the target location estimate at $j^{\text {th }}$ Monte Carlo trial and $J$ is the number of Monte Carlo trials. We observe that the performance of the proposed method approaches that of $\infty$-precision with increase in $M$. This implies that the proposed one-bit localization method is a proper choice for target localization in IoT systems where large number of radar nodes are always available.

In summary, the 1-bit sampling offers an attractive solution to the challenges posed by the NB-IoT for location-based services. The 1-bit samplers are integral to developing low cost and low power devices. We proposed a 1-bit passive sensor array formulation to estimate the ToA in an NB-IoT network. Our approach is helpful in addressing the problem of maintaining high localization accuracy while deploying reduced-rate ADCs at the nodes to conform with a reduced-bandwidth NB-IoT. Further, our novel method casts the ToA estimation algorithm as a multivariate fractional optimization problem and solves it via the Lasserre's SDP relaxation. This approach is computationally more efficient than the prior works and our numerical experiments demonstrated its feasibility.

\section{REFERENCES}

[1] E. Sisinni, A. Saifullah, S. Han, U. Jennehag, and M. Gidlund, "Industrial internet of things: Challenges, opportunities, and directions," IEEE Transactions on Industrial Informatics, vol. 14, no. 11, pp. 4724-4734, 2018.

[2] J. Xu, J. Yao, L. Wang, Z. Ming, K. Wu, and L. Chen, "Narrowband internet of things: Evolutions, technologies, and open issues," IEEE Internet of Things Journal, vol. 5, no. 3, pp. 1449-1462, 2017.

[3] W. Yang, M. Wang, J. Zhang, J. Zou, M. Hua, T. Xia, and X. You, "Narrowband wireless access for low-power massive internet of things: A bandwidth perspective," IEEE wireless communications, vol. 24, no. 3, pp. 138-145, 2017.

[4] F. Khelifi, A. Bradai, A. Benslimane, P. Rawat, and M. Atri, "A survey of localization systems in internet of things," Mobile Networks and Applications, vol. 24, no. 3, pp. 761-785, 2019.

[5] B. Kellogg, V. Talla, S. Gollakota, and J. R. Smith, "Passive Wi-Fi: 
Bringing low power to Wi-Fi transmissions," in USENIX Symposium on Networked Systems Design and Implementation, 2016, pp. 151-164.

[6] C. Pérez-Penichet, F. Hermans, A. Varshney, and T. Voigt, "Augmenting IoT networks with backscatter-enabled passive sensor tags," in ACM Workshop on Hot Topics in Wireless, 2016, pp. 23-27.

[7] J. F. Ensworth and M. S. Reynolds, "Every smart phone is a backscatter reader: Modulated backscatter compatibility with Bluetooth 4.0 Low Energy (BLE) devices," in IEEE International Conference on RFID, 2015, pp. 78-85.

[8] L. Zhang, Y. Liang, and M. Xiao, "Spectrum sharing for internet of things: A survey," IEEE Wireless Communications, vol. 26, no. 3, pp. 132-139, 2019.

[9] B. Kellogg, A. Parks, S. Gollakota, J. R. Smith, and D. Wetherall, "WiFi backscatter: Internet connectivity for RF-powered devices," $A C M$ SIGCOMM Computer Communication Review, vol. 44, no. 4, pp. 607618, 2015

[10] Q. Song, S. Guo, X. Liu, and Y. Yang, "CSI amplitude fingerprintingbased NB-IoT indoor localization," IEEE Internet of Things Journal, vol. 5, no. 3, pp. 1494-1504, 2017.

[11] S. Hu, A. Berg, X. Li, and F. Rusek, "Improving the performance of OTDOA based positioning in NB-IoT systems," in IEEE Global Communications Conference, 2017, pp. 1-7.

[12] S. Hu, X. Li, and F. Rusek, "On time-of-arrival estimation in NB-IoT systems," arXiv preprint arXiv:1711.03832, 2017.

[13] W. S. Jeon, S. B. Seo, and D. G. Jeong, "Effective frequency hopping pattern for ToA estimation in NB-IoT random access," IEEE Transactions on Vehicular Technology, vol. 67, no. 10, pp. 10 150-10 154, 2018.

[14] H. Sallouha, A. Chiumento, and S. Pollin, "Localization in long-range ultra narrow band IoT networks using RSSI," in IEEE International Conference on Communications, 2017, pp. 1-6.

[15] A. Noroozi and M. A. Sebt, "Target localization from bistatic range measurements in multi-transmitter multi-receiver passive radar," IEEE Signal Processing Letters, vol. 22, no. 12, pp. 2445-2449, 2015.

[16] P. T. Boufounos and R. G. Baraniuk, "1-bit compressive sensing," in Annual Conference on Information Sciences and Systems, 2008, pp. $16-21$.

[17] Z. Li, W. Xu, X. Zhang, and J. Lin, "A survey on one-bit compressed sensing: Theory and applications," Frontiers of Computer Science, vol. 12, no. 2, pp. 217-230, 2018.

[18] K. V. Mishra and Y. C. Eldar, "Sub-Nyquist radar: Principles and prototypes," in Compressed Sensing in Radar Signal Processing, A. D. Maio, Y. C. Eldar, and A. Haimovich, Eds. Cambridge University Press, 2019, in press.

[19] K. V. Mishra, M. R. Bhavani Shankar, V. Koivunen, B. Ottersten, and S. A. Vorobyov, "Toward millimeter wave joint radar communications: A signal processing perspective," IEEE Signal Processing Magazine, 2019 , in press.

[20] A. Zymnis, S. Boyd, and E. Candes, "Compressed sensing with quantized measurements," IEEE Signal Processing Letters, vol. 17, no. 2, pp. 149-152, 2009.

[21] K. Knudson, R. Saab, and R. Ward, "One-bit compressive sensing with norm estimation," IEEE Transactions on Information Theory, vol. 62, no. 5, pp. 2748-2758, 2016.

[22] M. Cho, K. V. Mishra, and W. Xu, "Computable performance guarantees for compressed sensing matrices," EURASIP Journal on Advances in Signal Processing, vol. 2018, no. 1, p. 16, 2018.

[23] H. Fu and Y. Chi, "Quantized spectral compressed sensing: CramérRao bounds and recovery algorithms," IEEE Transactions on Signal Processing, vol. 66, no. 12, pp. 3268-3279, 2018.

[24] C. Gianelli, L. Xu, J. Li, and P. Stoica, "One-bit compressive sampling with time-varying thresholds for sparse parameter estimation," in IEEE Sensor Array and Multichannel Signal Processing Workshop, 2016, pp $1-5$.

[25] O. Dabeer and A. Karnik, "Signal parameter estimation using 1-bit dithered quantization," IEEE Transactions on Information Theory, vol. 52, no. 12 , pp. 5389-5405, 2006.
[26] O. Dabeer and E. Masry, "Multivariate signal parameter estimation under dependent noise from 1-bit dithered quantized data," IEEE Transactions on Information Theory, vol. 54, no. 4, pp. 1637-1654, 2008.

[27] H. Chen and P. K. Varshney, "Performance limit for distributed estimation systems with identical one-bit quantizers," IEEE Transactions on Signal Processing, vol. 58, no. 1, pp. 466-471, 2009.

[28] X. Huang and B. Liao, "One-bit MUSIC," IEEE Signal Processing Letters, vol. 26, no. 7, pp. 961-965, 2019.

[29] O. Bar-Shalom and A. J. Weiss, "DOA estimation using one-bit quantized measurements," IEEE Transactions on Aerospace and Electronic Systems, vol. 38, no. 3, pp. 868-884, 2002.

[30] C.-L. Liu and P. Vaidyanathan, "One-bit sparse array DOA estimation," in IEEE International Conference on Acoustics, Speech and Signal Processing, 2017, pp. 3126-3130.

[31] C. Stöckle, J. Munir, A. Mezghani, and J. A. Nossek, "1-bit direction of arrival estimation based on compressed sensing," in IEEE International Workshop on Signal Processing Advances in Wireless Communications, 2015, pp. 246-250

[32] J. Mo and R. W. Heath, "Capacity analysis of one-bit quantized MIMO systems with transmitter channel state information," IEEE Transactions on Signal Processing, vol. 63, no. 20, pp. 5498-5512, 2015.

[33] H. Kim and J. Choi, "Channel estimation for one-bit massive MIMO systems exploiting spatio-temporal correlations," in IEEE Global Communications Conference, 2018, pp. 1-6.

[34] A. M. Elbir and K. V. Mishra, "Joint antenna selection and hybrid beamformer design using unquantized and quantized deep learning networks," arXiv preprint arXiv:1905.03107, 2019.

[35] H. Zayyani, M. Korki, and F. Marvasti, "Dictionary learning for blind one-bit compressed sensing," IEEE Signal Processing Letters, vol. 23, no. 2, pp. 187-191, 2015.

[36] Y. Chi and H. Fu, "Subspace learning from bits," IEEE Transactions on Signal Processing, vol. 65, no. 17, pp. 4429-4442, 2017.

[37] X. Dong and Y. Zhang, "A MAP approach for 1-bit compressive sensing in synthetic aperture radar imaging," IEEE Geoscience and Remote Sensing Letters, vol. 12, no. 6, pp. 1237-1241, 2015.

[38] K. V. Mishra, A. Kruger, and W. F. Krajewski, "Compressed sensing applied to weather radar," in IEEE Geoscience and Remote Sensing Symposium, 2014, pp. 1832-1835.

[39] B. Zhao, L. Huang, J. Li, M. Liu, and J. Wang, "Deceptive SAR jamming based on 1-bit sampling and time-varying thresholds," IEEE Journal of Selected Topics in Applied Earth Observations and Remote Sensing, vol. 11, no. 3, pp. 939-950, 2018.

[40] A. Ameri, J. Li, and M. Soltanalian, "One-bit radar processing and estimation with time-varying sampling thresholds," in IEEE Sensor Array and Multichannel Signal Processing Workshop, 2018, pp. 208212.

[41] Z. Cvetkovic, I. Daubechies, and B. F. Logan, "Single-bit oversampled A/D conversion with exponential accuracy in the bit rate," IEEE Transactions on Information Theory, vol. 53, no. 11, pp. 3979-3989, 2007.

[42] J. B. Lasserre, "Global optimization with polynomials and the problem of moments," SIAM Journal on Optimization, vol. 11, no. 3, pp. 796-817, 2001.

[43] H. Yanai, K. Takeuchi, and Y. Takane, Projection matrices, generalized inverse matrices, and singular value decomposition. Springer, 2011.

[44] C. D. Meyer, Matrix analysis and applied linear algebra. SIAM, 2000.

[45] W. Bruns and R. Schwänzl, "The number of equations defining a determinantal variety," Bulletin of the London Mathematical Society, vol. 22, no. 5, pp. 439-445, 1990.

[46] W. Bruns and U. Vetter, Determinantal rings, ser. Lecture Notes in Mathematics. Springer, 1988, vol. 1327. 